\title{
Critical roles for CCR2 and MCP-3 in monocyte mobilization from bone marrow and recruitment to inflammatory sites
}

\author{
Chia-Lin Tsou, ${ }^{1}$ Wendy Peters, ${ }^{1}$ Yue Si, ${ }^{1}$ Sarah Slaymaker, ${ }^{1}$ Ara M. Aslanian, ${ }^{1}$ \\ Stuart P. Weisberg, ${ }^{2}$ Matthias Mack, ${ }^{3}$ and Israel F. Charo,
}

\begin{abstract}
${ }^{1}$ Gladstone Institute of Cardiovascular Disease, UCSF, San Francisco, California, USA. ${ }^{2}$ Department of Medicine, Naomi Berrie Diabetes Center, Columbia University, New York, New York, USA. ${ }^{3}$ Department of Internal Medicine, Ludwig-Maximilians University of Munich, Munich, Germany. ${ }^{4}$ Cardiovascular Research Institute, Department of Medicine, UCSF, San Francisco, California, USA.
\end{abstract}

\begin{abstract}
Monocyte recruitment to sites of inflammation is regulated by members of the chemokine family of chemotactic cytokines. However, the mechanisms that govern the migration of monocytes from bone marrow to blood and from blood to inflamed tissues are not well understood. Here we report that CC chemokine receptor 2 (CCR2) is highly expressed on a subpopulation of blood monocytes whose numbers are markedly decreased in $\mathrm{CCR2}^{-/-}$mice. In bone marrow, however, $\mathrm{CCR}^{-/-}$mice had an increased number of monocytes, suggesting that CCR2 is critical for monocyte egress. Intravenous infusion of ex vivo-labeled WT or CCR2 ${ }^{-/-}$bone marrow into WT recipient mice demonstrated that CCR2 is necessary for efficient monocyte recruitment from the blood to inflamed tissue. Analysis of mice lacking monocyte chemoattractant protein-1 (MCP-1), MCP-3, MCP-5, or MCP-2 plus MCP-5 revealed that MCP-3 and MCP-1 are the CCR2 agonists most critical for the maintenance of normal blood monocyte counts. These findings provide evidence that CCR2 and MCP-3/MCP-1 are critical for monocyte mobilization and suggest new roles for monocyte chemoattractants in leukocyte homeostasis.
\end{abstract}

\section{Introduction}

Recruitment of monocytes to sites of inflammation is critical for host defense and is regulated by chemokines $(1,2)$. Among the most thoroughly characterized chemokines are the monocyte chemoattractant proteins (MCPs). MCPs attract cells through activation of their cognate receptor, CC chemokine receptor 2 (CCR2), which is expressed on the monocyte surface (3). Mice that are genetically deficient in CCR2 (CCR2 $2^{-/}$mice) exhibit markedly reduced tissue recruitment of monocytes in peritonitis (4), autoimmune encephalitis (5), tuberculosis (6), and atherosclerosis (7). Similar impairments in monocyte migration have been reported in mice lacking MCP-1 (CC chemokine ligand 2 [CCL2]), a major ligand for CCR2 $(8,9)$. Specific functions for the other murine MCPs (MCP-2, MCP-3/CCL7, and MCP-5/CCL12) have not been identified. Despite abundant evidence that CCR2 is critical for monocyte recruitment, the mechanisms that govern the movement of monocytes from bone marrow to blood and from blood to inflamed tissues are not well understood.

Recent studies have revealed considerable heterogeneity in circulating monocytes and identified inflammatory monocytes that are highly mobile and rapidly recruited to inflamed tissues (10-12). To determine whether specific chemokines and chemokine receptors differentially regulate the migration of inflammatory leukocytes, we examined monocytes in WT and CCR2-/mice. CCR $2^{-/-}$mice had a reduction in a subset of circulating blood monocytes and a concomitant increase in bone marrow monocytes and monocyte precursors.

Nonstandard abbreviations used: CCR2, CC chemokine receptor 2; MCP, monocyte chemoattractant protein; SDF- $\alpha$, stromal cell-derived factor $\alpha$.

Conflict of interest: I.F. Charo received research support in excess of $\$ 50,000$ from Daiichi Sankyo Inc. for work in the area of chemokine biology.

Citation for this article: J. Clin. Invest. 117:902-909 (2007). doi:10.1172/JCI29919.
To establish the relative contributions of individual MCP ligands to CCR2 activation and monocyte mobilization from the bone marrow, we analyzed the blood of MCP-1-deficient (MCP-1-/-) mice and newly created MCP-3-/-, MCP-5/-, and MCP-2-/-MCP-5/mice. In $\mathrm{MCP}-3^{-/-}$mice, the number of inflammatory monocytes was profoundly reduced, and a similar trend was seen in $\mathrm{MCP}-1^{-/}$ mice. These data establish important roles for MCP-3 and MCP-1 in CCR2-dependent blood monocyte homeostasis and mobilization from the bone marrow.

\section{Results}

FACS analysis of leukocyte subsets. To delineate subpopulations, peripheral blood mononuclear cells were harvested and incubated with antibodies specific to the surface markers 7/4 and Ly-6G. 7/4 detects a polymorphic $40-\mathrm{kDa}$ antigen expressed on neutrophils and monocytes (13). Ly-6G is a marker for polymorphonuclear leukocytes (14). By morphology, the $7 / 4^{\text {bri }} \mathrm{Ly}-6 \mathrm{G}^{+}$cells were neutrophils (Figure 1A). The Ly-6G- cells fell into 2 groups, $7 / 4^{\text {bri }}$ and $7 / 4^{\mathrm{dim}}$. The $7 / 4^{\text {bri }} \mathrm{Ly}-6 \mathrm{G}^{-}$cells were a homogeneous population of cells with monocyte morphology. The $7 / 4^{\text {dim } L y-6 G^{-}}$cells were a mixture of monocytes (50\%), T cells, B cells, and NK cells.

To further characterize these cell populations, we performed 4-color staining and quantified the expression of CCR2 and F4/80. The $7 / 4^{\text {bri }}$ Ly- $6 \mathrm{G}^{-}$cells were virtually $100 \%$ positive for both $\mathrm{F} 4 / 80$ (Figure 1B) and CCR2 (Figure 1C). The $7 / 4^{\mathrm{dim}}$ population was approximately $50 \%$ CCR 2 and $\mathrm{F} 4 / 80$ positive; the Ly- $6 \mathrm{G}^{+}$population was negative for both CCR2 and F4/80. Expression of CCR2 was significantly higher on the $7 / 4^{\text {bri }}$ than $7 / 4^{\mathrm{dim}}$ cells (Figure 1D).

Decrease in $7 / 4^{\text {bri }} L y-6 G^{-}$monocytes in CCR2 $2^{-/-}$mice. CCR2 $2^{-/}$mice had a marked reduction in the number of $7 / 4^{\text {bri }}$ Ly- $6 \mathrm{G}^{-}$monocytes compared with WT mice (Figure 2). A more modest, though statistically significant, reduction in the $7 / 4^{\mathrm{dim}}$ population was also seen and correlated with a reduction in the $\mathrm{F} 4 / 80^{+}$cells in this gate 


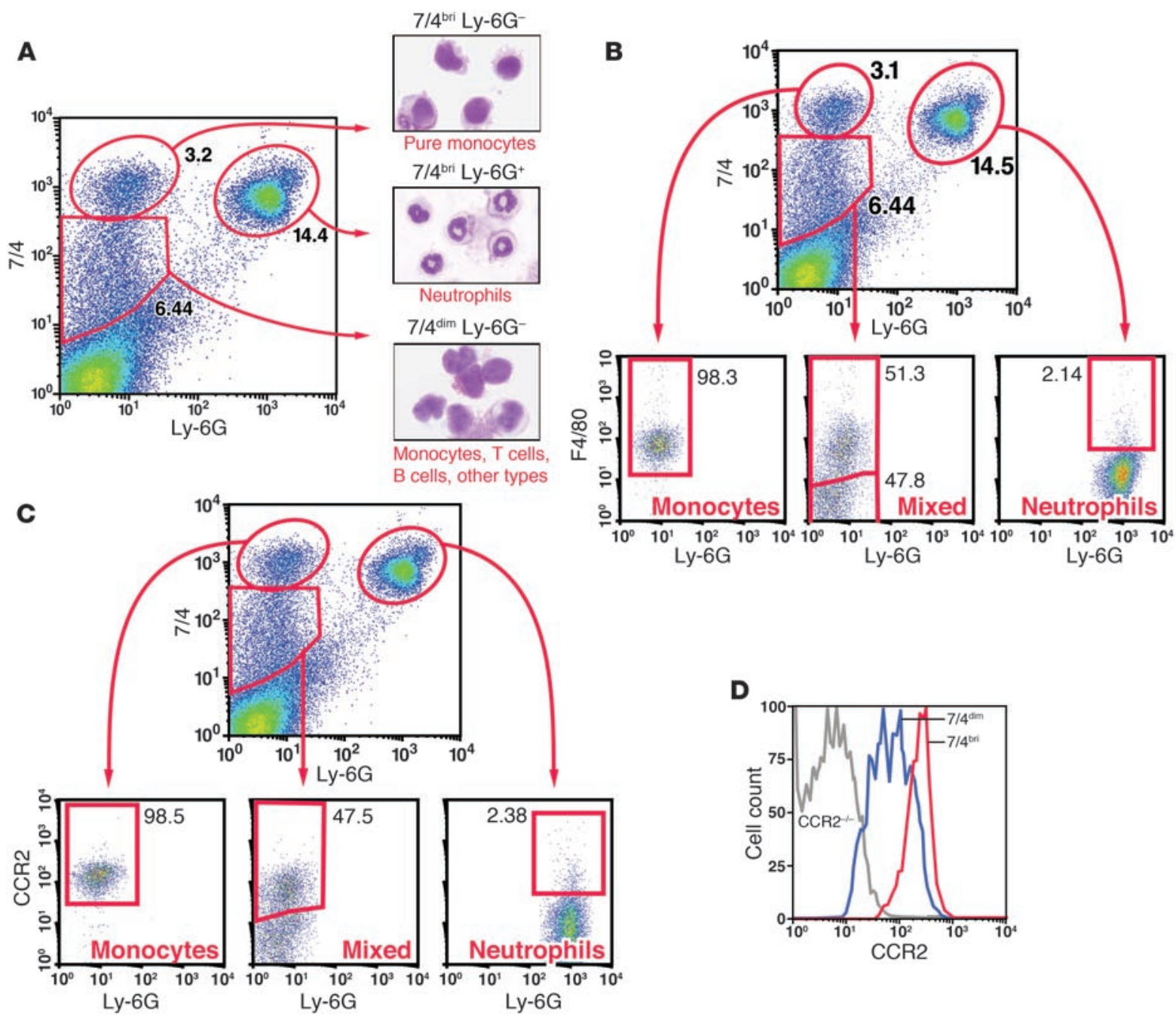

Figure 1

FACS analysis of murine peripheral blood leukocytes. (A) After lysis of red blood cells, leukocytes were stained with antibodies specific to $7 / 4$ and Ly-6G to reveal a population of pure monocytes (7/4briLy-6G-), a population of pure neutrophils $\left(7 / 4^{\text {bri }}\right.$ Ly- $\left.^{-} 6 \mathrm{G}^{+}\right)$, and a mixed population of monocytes, T cells, and B cells (7/4dim Ly-6G-). (B and C) Expression levels of F4/80 (B) and CCR2 (C) on populations of monocytes

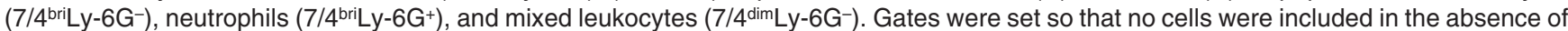
the primary antibody. Numbers are percentages of total leukocytes in each population. (D) Expression of CCR2 on monocytes (7/4briLy-6G-) and mixed leukocytes (7/4 $\left.\operatorname{dim} L y-6 G^{-}\right)$.

(data not shown). In WT mice, but not in CCR2-/- mice, a high-fat diet for 12 weeks induced significant monocytosis, particularly of $7 / 4^{\text {bri }}$ monocytes (Figure 3).

Failure of monocytes to exit the bone marrow in CCR2-/- mice. To determine whether CCR2 is critical for egress of monocytes or their precursors from the bone marrow, we placed apoE-/- mice on a high-fat Western diet for 3.5 weeks and analyzed leukocyte populations in the peripheral blood and bone marrow. The absence of CCR2 dramatically decreased the $7 / 4^{\text {bri }}$ monocyte population in the blood (Figure 4A), while simultaneously increasing the size of this population in the bone marrow (Figure 4B). WT and CCR2-/mice had similar rates of proliferation of monocyte progenitors (data not shown). We conclude from these data that CCR2 contributes to monocyte egress from the bone marrow and that the monocytopenia in CCR2 $2^{-/}$mice reflects the failure of these cells to exit the bone marrow.
Monocyte populations in MCP-deficient mice. CCR2 function depends upon activation by 1 or more members of the MCP family of chemokines. To examine monocyte populations in the absence of each of these chemokines, we created $\mathrm{MCP}-3^{-/}$, MCP-5/-, and MCP-2-/-MCP-5/- mice. All mice were born at the expected Mendelian ratios and developed normally. The completeness and specificity of the gene deletions were confirmed by quantitative real-time PCR (Figure 5).

To quantify monocyte populations in $\mathrm{MCP}^{-/-}$mice, we analyzed peripheral blood samples by FACS. MCP-1/-- mice had a significant reduction (50\%) in 7/4 ${ }^{\text {bri }}$ cells (Figure 6). There was an even more dramatic reduction (65\%) in the $\mathrm{MCP}-3^{-/-}$mice. In sharp contrast, $\mathrm{MCP}-5^{-/-}$and $\mathrm{MCP}-2^{-/-} \mathrm{MCP}-5^{-/-}$mice had essentially normal numbers of monocytes in both populations. Very similar results were seen in the $7 / 4^{\mathrm{dim}}$ cells (data not shown). Thus, MCP-3 and MCP-1 were the predominant CCR2 ligands required to 
A
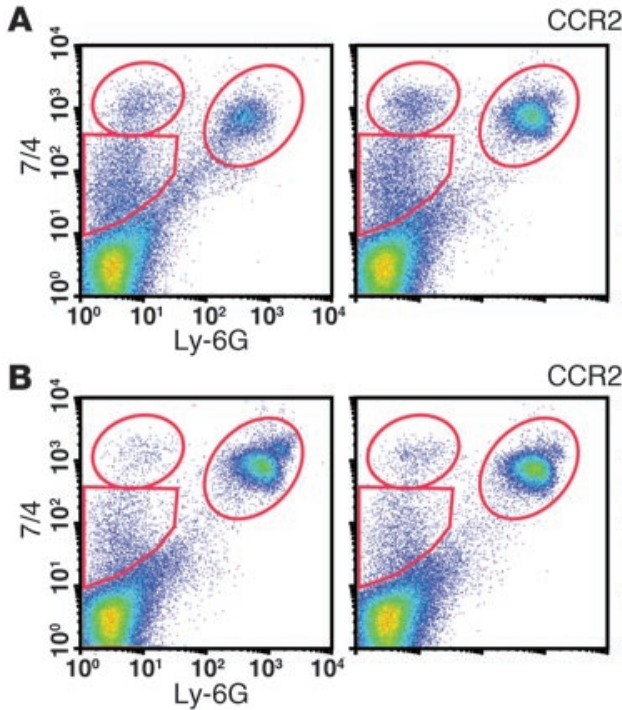

$\mathrm{CCR}^{-\prime}$
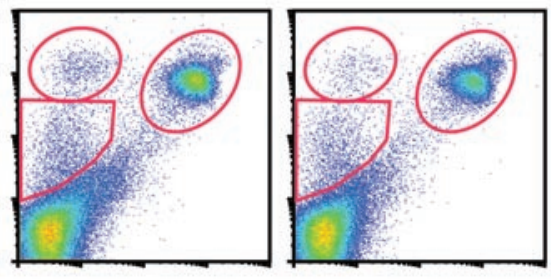

Figure 2

Monocytopenia in $\mathrm{CCR}^{-1-}$ mice. FACS plots of leukocytes obtained from $4 \mathrm{CCR} 2^{+/+}$mice (A) and 4 CCR2 ${ }^{-1-}$ mice (B) and stained for 7/4 and Ly-6G. (C) Quantification FACS plot data. Values are mean \pm SD. The total leukocyte count did not differ in CCR2 $2^{++}$and $\mathrm{CCR}^{-1-}$ mice. Typical absolute counts for the monocyte population (7/4briLy-6G-) were $2.7 \times 10^{5}$ for $\mathrm{CCR}^{+/+}$mice and $0.4 \times 10^{5}$ for $\mathrm{CCR}^{-1-}$ mice. For the mixed leukocyte populations (7/4dim Ly-6G-), the typical counts were $9.4 \times 10^{5}$ for $\mathrm{CCR}^{+/++}$mice and $3.5 \times 10^{5}$ for CCR2 ${ }^{-1-}$ mice. ${ }^{*} P<0.05$.

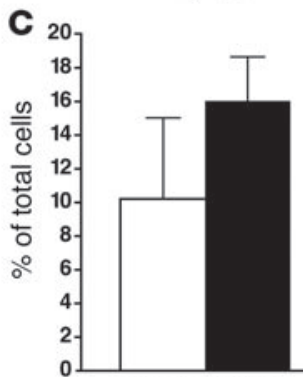

Neutrophils

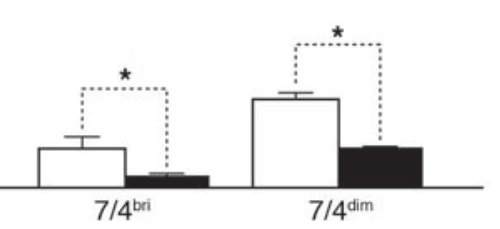

maintain normal numbers of blood monocytes. Consistent with this finding, examination of the bone marrow in mice challenged with intraperitoneal thioglycollate revealed an increase in the number of $7 / 4^{\text {bri }} \mathrm{Ly}-6 \mathrm{G}^{-}$cells in the CCR2 $2^{-/}$mice and $\mathrm{MCP}-3^{-/-}$ mice, and to a lesser extent in the MCP-1 $1^{-/}$mice, but not in the $\mathrm{MCP}-2^{-/-} \mathrm{MCP}-5^{-/-}$mice (Figure 7 ).

Adoptive transfer of $\mathrm{CCR} 2^{+/+}$versus $\mathrm{CCR} 2^{-/-}$bone marrow cells. To determine whether CCR2 was required for monocyte migration from the blood to tissues, as well as from the bone marrow to the blood, we quantified the monocyte populations in the peripheral blood and peritoneum 24 hours after instillation of thioglycollate to induce peritonitis. The number of $7 / 4^{\text {bri }}$ and $7 / 4^{\mathrm{dim}}$ cells in peripheral blood was significantly reduced in the absence of CCR2, but not in the absence of MCP-1 or MCP-3. Deletion of CCR2, MCP-1, or MCP-3, however, dramatically reduced monocyte

\section{Figure 3}

Effect of a high-fat diet on blood monocytes in CCR2 $2^{+/+}$ and $\mathrm{CCR}^{-1-}$ mice. Mice were fed regular laboratory chow or a high-fat Western diet for 45 weeks before sacrifice. (A) Typical FACS plots of total blood leukocytes obtained from $\mathrm{CCR}^{+/+}$and $\mathrm{CCR} 2^{-/-}$mice on the Western diet and stained for $7 / 4$ and Ly-6G as described in Figure 1. (B) Quantification of the FACS data showed an increase in the 7/4 bri (monocyte) population in CCR2 ${ }^{+/+}$, but not in CCR2 $2^{-/-}$, mice in response to the high-fat diet. The mixed leukocyte population $\left(7 / 4^{\mathrm{dim}}\right)$ had a more modest increase. Values are mean \pm SD. $n=4$ mice per genotype. ${ }^{*} P<0.05$.
A
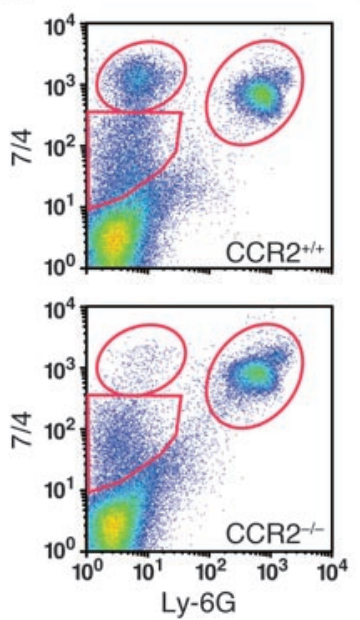

B

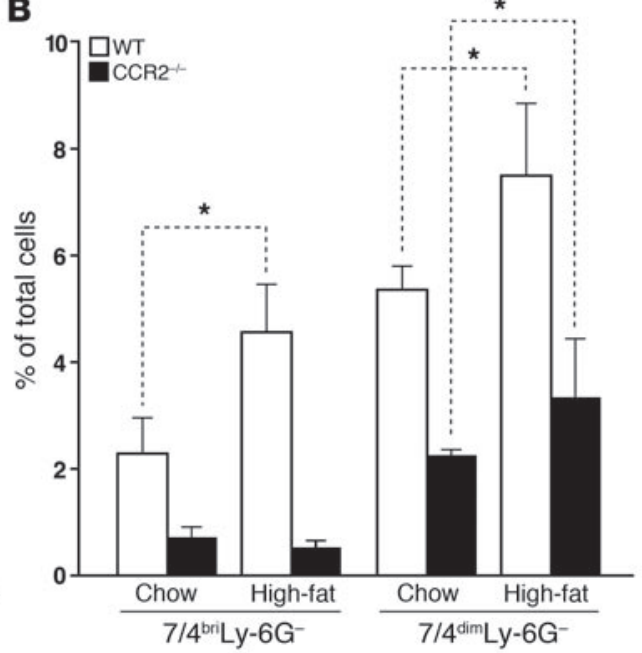


A
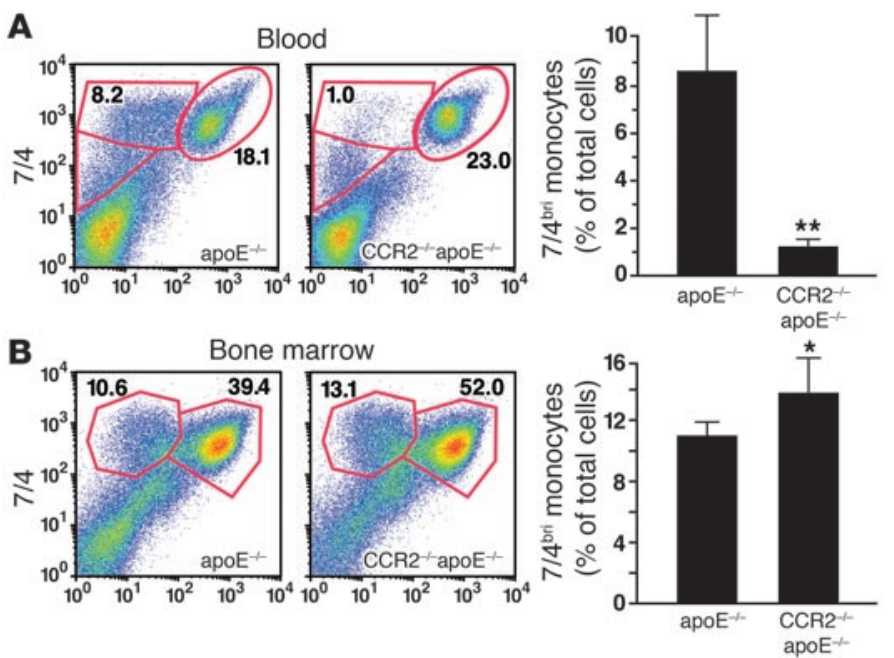

of thioglycollate into the peritoneum. Serum concentrations of MCP-1 and MCP-3 peaked at approximately 24 hours after instillation of thioglycollate and then fell back to baseline levels (Figure 8). At 66 hours after thioglycollate instillation, the peritoneal cells were harvested and analyzed by FACS. As seen in Figure 9, $\mathrm{CCR} 2^{+/+}$cells were recruited into the peritoneum more efficiently than CCR2 $-/$ cells. Taken together, these data indicate that CCR2 plays an important role in the directed migration of monocytes from the blood to inflamed tissues.

\section{Discussion}

Although CCR2 has a well-established role in recruiting monocytes to sites of inflammation, it has only recently been implicated in the mobilization of cells from the bone marrow to the peripheral circulation. This study shows that CCR2-/- mice have a marked decrease in a population of inflammatory blood monocytes, particularly

\section{Figure 4}

Monocyte retention in the bone marrow in $\mathrm{CCR}^{-/-}$mice. apo $\mathrm{E}^{-/-}$ mice were fed a high-fat Western diet for 3.5 weeks. (A) CCR2-/mice had fewer $7 / 4^{\text {bri }}$ monocytes in the blood than CCR2 ${ }^{+/+}$mice. ${ }^{* *} P<0.01 ; n=4$ mice per group. (B) Bone marrow cells were recovered and stained with $7 / 4$ and Ly-6G. CCR2 ${ }^{-1-}$ mice had an increase in the $7 / 4$ bri population. Error bars represent SD. ${ }^{\star} P<0.05 ; n=4$ mice per group.

in settings conducive to monocytosis, such as hypercholesterolemia. The bone marrow of CCR2 $2^{-/-}$mice contained normal or increased numbers of monocytes and monocyte progenitors, suggesting a defect in mobilization rather than monocyte differentiation. The chemokines that were primarily responsible for CCR2 activation and monocyte mobilization from the bone marrow were MCP-3 and MCP-1. These findings reveal an important role for CCR2 in the mobilization of hematopoietic cells from the bone marrow and provide what we believe to be the first evidence for a function of the chemokine MCP-3.

Monocytes and macrophages are highly heterogeneous populations of cells, and it has been difficult to identify surface markers that are monocyte specific. Recent efforts have used combinations of markers in an attempt to distinguish monocytes from macrophages, neutrophils, and other leukocytes and to identify functional subsets of monocytes $(10-12,15)$. CD11b is highly expressed on monocytes but is also abundant on neutrophils, eosinophils, and subsets of macrophages and dendritic cells (16, 17). F4/80 is a well-characterized macrophage marker that is expressed at lower levels on monocytes and can also be detected on eosinophils and dendritic cells (18). Similarly, the monocyte colony-stimulating factor receptor CD115 is expressed on monocytes, macrophages, and dendritic cells $(19,20)$. The Gr-1 antigen is composed of 2 antigens, Ly-6G and Ly-6C and is expressed on neutrophils, monocytes, and subsets of macrophages, $\mathrm{T}$ cells, and plasmacytoid dendritic cells (17). Although Gr-1 is often used to

\section{Figure 5}

Relative abundance of $\mathrm{MCPs}$ in $\mathrm{MCP}-1^{-/-}$ mice and newly created $\mathrm{MCP}-3^{--}, \mathrm{MCP}^{-} 5^{-1-}$, and $\mathrm{MCP}-2^{-/-} \mathrm{MCP}_{-} 5^{-/}$mice. The mice were immunized with keyhole limpet hemocyanin/CFA, and MCP mRNA levels in the draining lymph node were quantified by real-time PCR to confirm the specificity and completeness of the gene deletion.
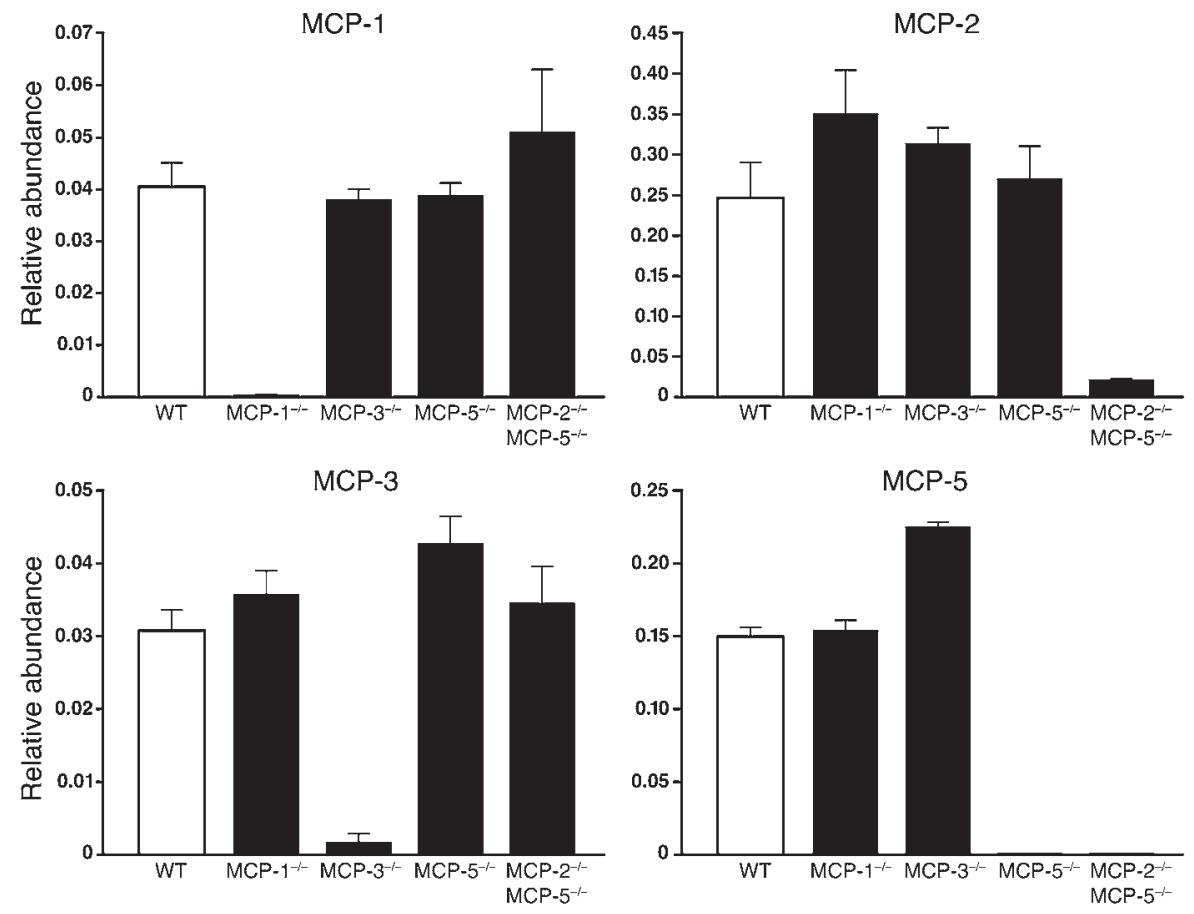


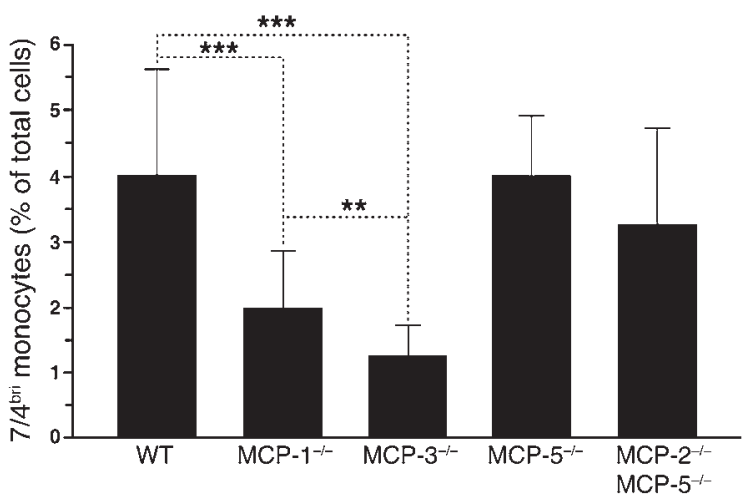

identify neutrophils, it is the Ly-6G component of Gr-1 that is almost exclusively neutrophil specific; the staining of other cell types by $\mathrm{Gr}-1$ is thus attributable to its Ly-6C antigen component (17). The polymorphic antigen $7 / 4$ is highly expressed on neutrophils, activated macrophages, and monocytes. Recent work by Taylor et al. (17) and Henderson et al. (10) has shown that $7 / 4^{\text {bri }} \mathrm{Gr}-1^{\text {int }}$ leukocytes are $\mathrm{F} 4 / 80^{+}$monocytes. The $7 / 4$ antigen is downregulated as monocytes differentiate into macrophages, suggesting that $7 / 4^{\text {bri }} \mathrm{Gr}-1^{\text {int }} \mathrm{F} 4 / 80^{+}$cells at sites of inflammation are newly arrived monocytes. This population of monocytes has therefore been referred to as inflammatory monocytes $(10,12,17)$.

Using a combination of Ly-6G and 7/4, we identified a homogenous population of monocytes that were $7 / 4^{\text {bri }} \mathrm{Ly}-6 \mathrm{G}^{-}$. This population was uniformly positive for F4/80 and CCR2 and was phenotypically identical to the inflammatory monocytes described by Henderson et al. as $7 / 4^{+} \mathrm{Gr}-1^{\text {int }}(10)$, the Ly- $6 \mathrm{C}^{\text {hi }}$ monocyte population described by Drevets et al. (15), and the Gr-1 ${ }^{\text {hi }}$ monocyte described by Tacke et al. (21). The use of neutrophil-specific Ly-6G

\section{Figure 6}

Decrease in the level of $7 / 4^{\text {briLy-6G- }}$ monocytes in MCP-3 ${ }^{-/-}$and $\mathrm{MCP}-1^{-1-}$ mice. Values in bar graphs are mean \pm SD. Data from 10 independent experiments are combined. WT $(n=66)$, MCP-1-1$(n=21), \mathrm{MCP}_{-} 3^{-/-}(n=17), \mathrm{MCP}^{-5^{-/-}}(n=10), \mathrm{MCP}^{-2^{-/} \mathrm{MCP}-5^{-/-}}$ $(n=12) .{ }^{\star \star} P<0.01 ;{ }^{\star \star \star} P<0.001$.

marker instead of the polymorphic Gr-1 antibody resulted in more clearly defined populations of cells in the FACS plots. We also identified a second population of cells that were $7 / 4^{\mathrm{dim}} \mathrm{Ly}-6 \mathrm{G}^{-}$. This heterogeneous population consisted of approximately $50 \%$ monocytes (defined as $\mathrm{F} 4 / 80^{+}$and CCR2 $2^{+}$), 20\%-30\% B cells (CD19+ ${ }^{+}$, F4/80-, CCR2-), and $10 \%-20 \% \mathrm{~T}$ cells $\left(\mathrm{CD}^{+}, \mathrm{F} 4 / 80^{-}\right)$. The $7 / 4^{\mathrm{dim}} \mathrm{Ly}^{-6 \mathrm{G}^{-}}$ monocytes likely correspond to the GFPhi $\mathrm{Gr}-1^{-}$monocytes described by Geissmann et al. as CX3CR1 ${ }^{\text {hi }}$ (22). Interestingly, CCR2 expression was lower in $7 / 4^{\mathrm{dim}}$ than in $7 / 4^{\text {bri }}$ monocytes.

Analysis of the blood of unchallenged CCR2 $2^{-/}$mice revealed a striking decrease in the size of the $7 / 4^{\text {bri }}$ population and a more modest but statistically significant decrease in the $7 / 4 \mathrm{dim}$ population, suggesting that CCR2 is required for normal monocyte homeostasis. To determine whether monocyte mobilization in response to inflammatory insults also requires CCR2, we fed the mice a high-fat, high-cholesterol diet for 3.5 weeks to induce hypercholesterolemia, which leads to reproducible monocyte/ macrophage accumulation in blood vessel walls and inflammation $(23,24)$. Others have reported an increase in the blood monocyte count in both mice (25) and humans (26) in response to chronic consumption of a high-fat diet. We found monocytosis in the $7 / 4^{\text {bri }}$ and $7 / 4^{\mathrm{dim}}$ populations in WT mice, but not in the CCR2 $2^{-/-}$ mice. Thus, CCR2 appears to be important in the mobilization of monocytes from the bone marrow to the blood under normal homeostatic conditions and in response to inflammation.
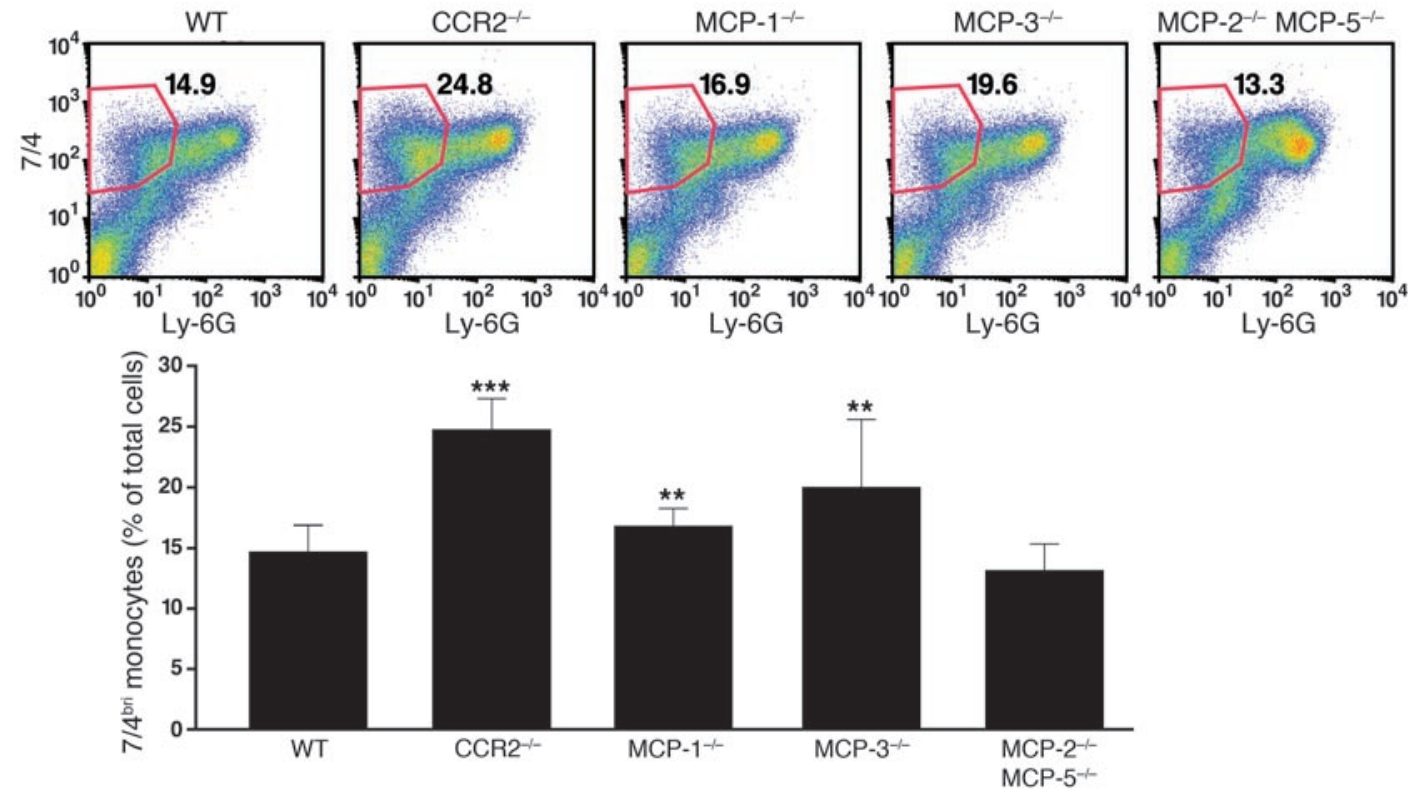

Figure 7

Monocyte retention in the bone marrow in $\mathrm{MCP}-1^{-1-}$ and $\mathrm{MCP}-3^{-/-}$mice. Mice received intraperitoneal thioglycollate. Bone marrow cells were harvested 1 day later and stained with $7 / 4$ and Ly-6G. The FACS gate was established by staining total bone marrow from WT mice for CCR2 ${ }^{+}$cells and then copying the gate onto the $7 / 4-$ versus-Ly-6G plot. WT $(n=8)$, MCP- $1^{-/-}(n=8), \mathrm{MCP}_{-} 3^{-/-}(n=8)$, MCP-2 $2^{-/-} \mathrm{MCP}-5^{-/-}$ $(n=9) .{ }^{* *} P<0.01 ;{ }^{* * *} P<0.001$. 


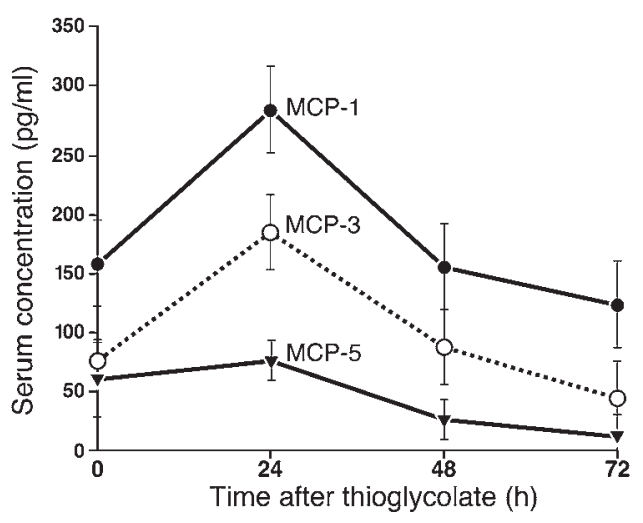

Figure 8

Transient rise in serum chemokine levels following intraperitoneal instillation of thioglycollate. Thioglycollate was instilled into the peritoneal cavity of the mice, and serum concentrations of MCP-1, MCP-3, and MCP- 5 were quantified by ELISA at the indicated time points. Results are typical of 2 experiments with 4-5 mice per genotype. Error bars represent SD.

Chemoattractants also play a role in mobilizing neutrophils. The complement fragment $\mathrm{C} 5 \mathrm{a}$, a potent neutrophil activator, induces neutrophil leukocytosis (27), as does infusion of IL-8 (28), an agonist of the neutrophil CXCR2 receptor. More recently, attention has focused on neutrophil CXCR4 and its agonist, stromal cell-derived factor $\alpha$ (SDF- $\alpha$ ). Infusion of CXCR4-blocking antibodies or small-molecule CXCR4 antagonists markedly increased the blood neutrophil count. SDF- $\alpha$ and CXCR4 also appear to be involved in the retention of neutrophils in the bone marrow and in the homing of circulating neutrophils back to the bone marrow $(29,30)$. Therefore, activation of CXCR 4 by SDF- $\alpha$ may be important in the retention of neutrophils in the bone marrow. In contrast, our findings suggest that CCR2 is required for monocyte egress from the bone marrow.

Although the importance of MCP-1 in monocyte recruitment and activation is well documented (31), relatively little is known about the physiological roles of MCP-2, MCP-3, or MCP-5. Whether these other MCPs are simply redundant CCR2 agonists or instead fulfill specific functions has been unclear. To address this question, we used homologous recombination in embryonic stem cells to target and delete these MCPs. MCP-5/-, $\mathrm{MCP}-3^{-/}$, and MCP-2-/-MCP-5 ${ }^{-/-}$ mice were completely fertile and developed normally. $\mathrm{MCP}-3^{-/-}$and MCP-1/- mice had decreases in the number of $7 / 4^{\text {bri }}$ monocytes but the deficit was significantly greater in the $\mathrm{MCP}-3^{-/}$group. Further, only $\mathrm{MCP}-1^{-/-}$and $\mathrm{MCP}-3^{-/-}$mice had deficits in monocyte/macrophage recruitment to the peritoneum in response to thioglycollateinduced inflammation (Supplemental Figure 1). Analysis of bone marrow from these mice revealed an increase in the $7 / 4^{+}$population in $\mathrm{MCP}-1^{-/-}$and $\mathrm{MCP}-3^{-/-}$mice, but not in $\mathrm{MCP}-2^{-/-} \mathrm{MCP}-5^{-/-}$mice. These findings reveal a new role for MCP-3 and suggest that MCP-1 and MCP-3 work in concert to regulate monocyte mobilization and recruitment to inflamed tissue.

The monocytopenia in the CCR2-/- mice raises the question of whether the dominant phenotype of these mice - reduced recruitment of monocyte/macrophages to sites of inflammation and injury - can be solely attributed to the reduced number of CCR $2^{+}$ monocytes in the blood, rather than to a failure of monocytes to move from the blood into inflamed tissues. Serbina and Pamer
(32) presented evidence for the former scenario and argued that, in the setting of infection with monocytogenes, CCR2 $2^{-/}$and $\mathrm{CCR} 2^{+/+}$monocytes are recruited equally well from blood to inflamed tissues. To directly compare the ability of CCR2 $2^{+/+}$and CCR2 $2^{-/}$monocytes to migrate into tissues, we performed adoptive transfer of CFSE-labeled bone marrow cells. Because the thioglycollate-induced increase in the MCP levels peaks at 24 hours and then rapidly returns to baseline, we performed the intravenous adoptive transfer and intraperitoneal instillation of thioglycollate simultaneously. Under these conditions CCR2 $2^{+/+}$monocytes were recruited into the peritoneum much more efficiently than CCR2 $2^{--}$monocytes (Figure 9). We have performed the adoptive transfer of monocytes approximately 60 hours after instillation of thioglycollate, as was done by Serbina and Pamer (32), and find that recruitment at this time point is CCR2 independent (data not shown). Taken together, these results suggest that the early phase of monocyte migration to the inflamed peritoneum is CCR2 dependent, whereas later phases are CCR2 independent. The chemoattractants mediating late-phase migration are not known but might include other chemokines, complement fragments, or arachidonic acid metabolites.

In summary, we have shown that CCR 2 and MCP-3 are critical for the maintenance of monocyte homeostasis. Surface expression of CCR2 marks a subpopulation of circulating monocytes and is necessary for their efficient release from the bone marrow. Whether or not CCR2 or MCP-3 plays a role in the recirculation of these monocytes through the bone marrow is yet to be determined. Similarly, it will be important to determine whether activation of CCR1 or CCR3, the other 2 chemokine receptors for MCP-3, contributes to monocyte recruitment. Our findings reveal important roles for CCR2 and MCP-3 in monocyte homeostasis under resting conditions and in the monocytic response to inflammatory stimuli.
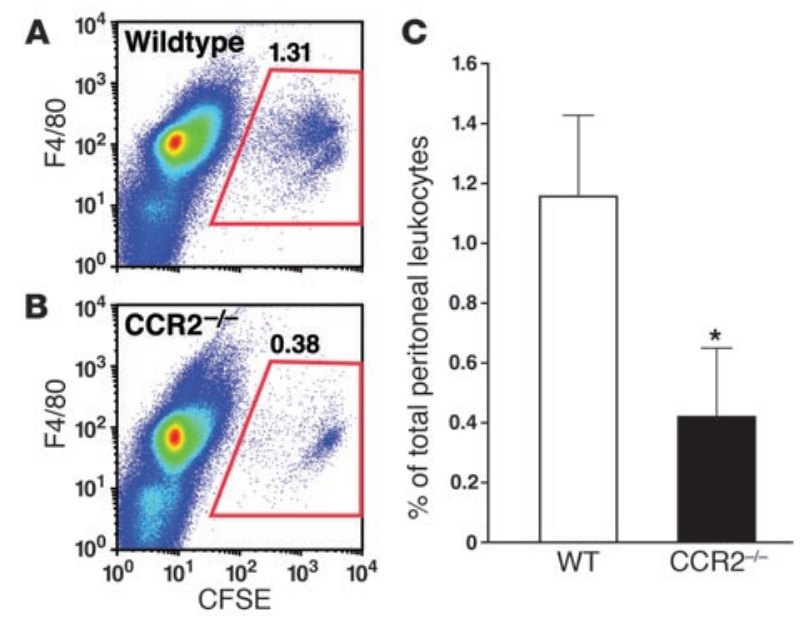

\section{Figure 9}

Adoptive transfer of bone marrow cells. Bone marrow was harvested from $\mathrm{CCR}_{2}^{+/+}$and $\mathrm{CCR}^{-/-}$mice, labeled with CFSE, and infused intravenously into WT recipients. Thioglycollate was instilled into the peritoneal cavity of the recipient mice coincident with the bone marrow infusion, and the peritoneal cells were harvested 66 hours later. FACS plots of $\mathrm{F} 4 / 80^{+} \mathrm{CFSE}+$ leukocytes from mice infused with labeled $\mathrm{CCR}^{+/+}(\mathbf{A})$ and $\mathrm{CCR}^{-/-}(\mathbf{B})$ bone marrow. (C) Quantification of FACS plots. Values are mean \pm SD of the total number of peritoneal leukocytes present. $n=4$ mice per genotype; ${ }^{*} P<0.05$ versus WT. Results are typical of 2 experiments. 


\section{Methods}

Mice. CCR2 $2^{-/-}$mice and MCP-1/-- mice were generated as described previously $(4,8)$ and backcrossed for 10 generations onto a C57BL/ 6 background. For generation of the MCP-3/- mice, a mouse $129 / \mathrm{Sv}$ strain bacterial artificial chromosome library (RPCI-22.C; Research Genetics Inc.) was screened using a probe generated by PCR specific for the coding region of $M C P-3$. Three positive clones were obtained and used to generate arms for targeting vector construction. Flanking regions of the MCP-3 gene were amplified by PCR with an Expand High Fidelity Kit (Roche Applied Science). An upstream region of 4,587 bp was generated with primers containing BamH1 restriction sites indicated by underlining (5' -CGGGATCCTGTGGAGTGATGAAATGGGGG-3' and 5'CGGGATCCCAGGAGAGTGTAATGTGAGGCTGG-3'). A downstream region of 3,172 bp was generated with primers containing PacI and NotI restriction sites indicated by underlining (5'-CCTTAATTAATGAACAGGGTGGCAAGAAGTAGG-3' and 5' -ATAAGAATGCGGCCGCTCAGCAGCAATCCCATAGCC-3').

To remove the coding region of $\mathrm{MCP}-3$, the amplicons were ligated into a pSV-Neo-TK vector. The MCP-3 targeting vector was linearized with NotI, electroporated into RF8 embryonic stem cells, and selected with G418 and 1-(2-deoxy-2-fluoro- $\beta$-D-arabinofuranosyl)-5-iodouracil (FIAU). Colonies were screened by PCR with primers located within the neo cassette and downstream of the $3^{\prime}$ targeting arm (5'-TTCCTCGTGCTTTACGGTATCG-3' and 5'-AGAAGTTTCCTCACAGACACCTGC-3'). The resulting amplicon was 4,132 bp in correctly targeted clones. To confirm recombination by Southern blotting, BamH1-digested genomic DNA was hybridized with a $0.8-\mathrm{kb}$ probe specific to a region $5^{\prime}$ of the upstream targeting arm.

MCP-3 $3^{-/-}$mice had no obvious developmental abnormalities and were born at the expected Mendelian ratios. To confirm the absence of MCP-3 mRNA, mice were immunized with keyhole limpet hemocyanin/CFA, and MCP levels in the draining lymph node were quantified by real-time PCR. MCP-3 mRNA was undetectable in MCP-3-/- mice, whereas MCP-1, MCP-2, and MCP-5 mRNAs were present at WT levels (Figure 5).

To create $\mathrm{MCP}-5^{-/-}$mice, a mouse bacterial artificial chromosome, clone CITB-CJ7-B, containing the MCP-5 gene (Research Genetics Inc.) was digested, and MCP-5 fragments were subcloned for sequencing. Approximately $14 \mathrm{~kb}$ of the surrounding MCP-5 genomic locus was sequenced. From this sequence information, primers were designed to amplify the 2.3-kb short arm from the EcoRI fragment and the 5.9-kb long arm from the BamHI fragment. The Long PCR kit was used (Roche Applied Science), and using primer-encoded restriction sites, the short and long arms were cloned into the pSV-Neo-TK vector, with EcoI for the short arm and FseI for the long arm. The MCP-5 targeting vector was linearized with XhoI, electroporated into RF8 embryonic stem cells, and cultured on leukemiainhibiting factor-producing STO feeder cells.

Clones resistant to both G418 $(150 \mu \mathrm{g} / \mathrm{ml})$ and FIAU $(0.25 \mu \mathrm{M})$ were screened by Southern blot analysis of HindIII-digested genomic DNA hybridized with a probe located $5^{\prime}$ of the targeting vector. This Southern blot assay was also used for screening the mouse lines. In addition, a PCR assay was used to screen mouse lines containing a common 5' primer, AGACTCTGAGGAATGACCTTGCC, and two 3' primers, AGACTGCCTTGGGAAAAGCG (neo gene) and CCAATACCTGACGACTGATGGTGG (MCP-5 coding region).

Analysis of chemokine mRNA in these mice revealed that in addition to MCP-5 mRNA, MCP-2 mRNA (the MCP-2 gene is located approximately $12 \mathrm{~kb}$ upstream of the MCP-5 gene) was also essentially absent (Figure 5), indicating that the mice were MCP-2-/-MCP-5/- double-knockout. Other MCP mRNAs were present in normal amounts in these mice. The absence of MCP-2 was due to expression of the nearby neomycin resistance gene; mating the MCP-2 $2^{-/} \mathrm{MCP}-5^{-/-}$mice with Cre-deleter mice restored expression of MCP-2 mRNA, resulting in pure MCP-5//- mice (Figure 5).
All mice were housed and bred with littermate controls in specific pathogen-free conditions and were studied at 6-12 weeks of age. All animal experiments were approved by the UCSF Animal Care Committee. All mice were at least $96.87 \%$ C57BL/6 as determined by MAX-BAX marker-assisted backcrossing analysis (Charles River Laboratories).

Analysis of monocyte subsets. Blood was collected from the abdominal aorta of anesthetized mice and depleted of red cells by lysis buffer $(0.15 \mathrm{M}$ $\mathrm{NH}_{4} \mathrm{Cl}, 1 \mathrm{mM} \mathrm{KHCO}, 0.1 \mathrm{mM}$ EDTA). Leukocytes $\left(3 \times 10^{5}\right)$ were washed in staining buffer (PBS, 1\% FCS, 0.09\% sodium azide) and incubated with rat anti-mouse CD16/CD32 (1:500, or $1 \mu \mathrm{g} / \mathrm{ml}$; BD Biosciences) for 30 minutes to block Fc receptors. Leukocytes were incubated for $30 \mathrm{~min}$ utes at $4{ }^{\circ} \mathrm{C}$ with fluorescently labeled antibodies: Ly-6G-FITC (1:200, or $2.5 \mu \mathrm{g} / \mathrm{ml}$; BD Biosciences), 7/4-PE (1:50; Serotec), F4/80-APC (1:1,000, or $0.2 \mu \mathrm{g} / \mathrm{ml}$; CALTAG Laboratories), CD3-biotin (1:200; BD Biosciences), CD-19-perCPCy5.5 (1:1,000; BD Biosciences). The cells were then washed twice with staining buffer and stored at $4^{\circ} \mathrm{C}$. Flow cytometry was performed on a FACSCalibur (BD Biosciences). Data were acquired with CellQuest Pro software (version 5.1.1; BD Biosciences) and analyzed with FlowJo software (version 6.4.7; Tree Star). The Zebra plot feature of FlowJo was used to set the gates, based on unstained samples.

The antibody against murine CCR2 has been described previously (33). Cytospins were stained with modified Wright stain (Sigma-Aldrich). Bone marrow was flushed from the tibias and femurs of mice with HBSS supplemented with $1 \%$ FBS and 10 mM HEPES, and red blood cells were depleted using lysis buffer. Bone marrow cells were washed with HBSS plus 1\% FCS plus $10 \mathrm{mM}$ HEPES and incubated with Fc blocker for 30 minutes. Flow cytometry was performed as detailed above. In some experiments, mice were fed a high-fat Western diet (45\% of total calories from fat; D12451; Research Diets Inc.).

Thioglycollate-induced peritonitis. Peritonitis was induced with thioglycollate in $\mathrm{CCR} 2^{-/-}$mice and $\mathrm{MCP}-1^{-/-}$mice as described previously (4). Peritoneal leukocytes were collected 72 hours later by flushing the peritoneal cavity with $10 \mathrm{ml}$ of PBS. After red blood cell lysis, peritoneal cells were washed with staining buffer, and $3 \times 10^{6}$ cells were stained with mouse anti-mouse CD16/CD32 (1:50; CALTAG Laboratories) for 30 minutes to block Fc receptors. After washing to ensure removal of excess secondary antibody, the peritoneal cells were incubated simultaneously with F4/80 PE-Cy7 (1:100; eBioscience) and streptavidin-APC $(1: 1,000)$ (BD Biosciences) for 30 minutes. Cells were washed twice with staining buffer and analyzed by flow cytometry, as described above.

Adoptive transfer and flow cytometry of peritoneal leukocytes. Bone marrow cells were harvested from the femur and tibia of WT and CCR2-/- mice; red blood cells were depleted by ACK lysis buffer $\left(0.15 \mathrm{M} \mathrm{NH}_{4} \mathrm{Cl}, 1 \mathrm{mM}\right.$ $\mathrm{KHCO}_{3}, 0.1 \mathrm{mM}$ EDTA); and the remaining cells were washed with PBS and resuspended at a density of $3 \times 10^{7}$ to $5 \times 10^{7}$ cells $/ \mathrm{ml}$ in PBS containing $2 \mu \mathrm{M}$ CFSE (Molecular Probes; Invitrogen). The cell suspension was incubated at $37^{\circ} \mathrm{C}$ for 10 minutes, washed 3 times with cold PBS, and injected intravenously into WT recipient mice. Each recipient mouse received $2.5 \times 10^{7}$ to $3.0 \times 10^{7}$ cells. To induce inflammation, $3 \mathrm{ml}$ of $3 \%$ thioglycollate solution was instilled into the peritoneum at the time of bone marrow transfer.

Statistics. Data were analyzed using GraphPad Insert version 3.0. A nonparametric Mann-Whitney $U$ test and an unpaired 2-tailed Student's $t$ test were used. A $P$ value of less than 0.05 was considered significant.

\section{Acknowledgments}

This work was funded in part by NIH grants 5R01 HL52773-12 and R01 HL63894-07 (to I.F. Charo). We thank Gary Howard and Stephen Ordway for editorial assistance, Mijoung Chang for manuscript preparation, and John Carroll for preparation of the figures. 
Received for publication July 31, 2006, and accepted in revised form January 24, 2007.

Address correspondence to: Israel F. Charo, Gladstone Institute of Cardiovascular Disease, 1650 Owens Street, San Francisco, Cali-

1. Charo, I.F., and Ransohoff, R.M. 2006. The many roles of chemokines and chemokine receptors in inflammation. N. Engl. J. Med. 354:610-621.

2. Gerard, C., and Rollins, B.J. 2001. Chemokines and disease. Nat. Immunol. 2:108-115.

3. Charo, I.F., et al. 1994. Molecular cloning and functional expression of two monocyte chemoattractant protein 1 receptors reveals alternative splicing of the carboxyl-terminal tails. Proc. Natl. Acad. Sci. U. S. A. 91:2752-2756.

4. Boring, L., et al. 1997. Impaired monocyte migration and reduced type 1 (Th1) cytokine responses in C-C chemokine receptor 2 knockout mice. J. Clin. Invest. 100:2552-2561.

5. Izikson, L., et al. 2000. Resistance to experimental autoimmune encephalomyelitis in mice lacking the CC chemokine receptor (CCR)2. J. Exp. Med. 192:1075-1080.

6. Peters, W., et al. 2001. Chemokine receptor 2 serves an early and essential role in resistance to $\mathrm{Myco}$ bacterium tuberculosis. Proc. Natl. Acad. Sci. U. S. A. 98:7958-7963.

7. Boring, L., Gosling, J., Cleary, M., and Charo, I.F. 1998. Decreased lesion formation in CCR2 $2^{-/}$mice reveals a role for chemokines in the initiation of atherosclerosis. Nature. 394:894-897.

8. Lu, B., et al. 1998. Abnormalities in monocyte recruitment and cytokine expression in monocyte chemoattractant protein 1-deficient mice. J. Exp. Med. 187:601-608.

9. Huang, D., Wang, J., Kivisakk, P., Rollins, B.J., and Ransohoff, R.M. 2001. Absence of monocyte chemoattractant protein 1 in mice leads to decreased local macrophage recruitment and antigen-specific $\mathrm{T}$ helper cell type 1 immune response in experimental autoimmune encephalomyelitis. J. Exp. Med. 193:713-725.

10. Henderson, R.B., Hobbs, J.A., Mathies, M., and Hogg, N. 2003. Rapid recruitment of inflammatory monocytes is independent of neutrophil migration. Blood. 102:328-335.

11. Sunderkotter, C., et al. 2004. Subpopulations of mouse blood monocytes differ in maturation fornia 94158, USA. Phone: (415) 734-2000; Fax: (415) 355-0960; E-mail: icharo@gladstone.ucsf.edu.

Ara M. Aslanian's present address is: Novartis Institutes for BioMedical Research, Cambridge, Massachusetts, USA. stage and inflammatory response. J. Immunol. 172:4410-4417.

12. Gordon, S., and Taylor, P.R. 2005. Monocyte and macrophage heterogeneity. Nat. Rev. Immunol. 5:953-964.

13. Hirsch, S., and Gordon, S. 1983. Polymorphic expression of a neutrophil differentiation antigen revealed by monoclonal antibody $7 / 4$. Immunogenetics. 18:229-239.

14. Fleming, T.J., Fleming, M.L., and Malek, T.R. 1993. Selective expression of Ly-6G on myeloid lineage cells in mouse bone marrow. RB6-8C5 mAb to granulocyte-differentiation antigen (Gr-1) detects members of the Ly-6 family. J. Immunol. 151:2399-2408.

15. Drevets, D.A., et al. 2004. The Ly-6Chigh monocyte subpopulation transports Listeria monocytogenes into the brain during systemic infection of mice. J. Immunol. 172:4418-4424.

16. Lemos, M.P., Esquivel, F., Scott, P., and Laufer, T.M. 2004. MHC class II expression restricted to CD8alpha+ and CD11b+ dendritic cells is sufficient for control of Leishmania major. J. Exp. Med. 199:725-730.

17. Taylor, P.R., Brown, G.D., Geldhof, A.B., MartinezPomares, L., and Gordon, S. 2003. Pattern recognition receptors and differentiation antigens define murine myeloid cell heterogeneity ex vivo. Eur. J. Immunol. 33:2090-2097.

18. Taylor, P.R., et al. 2005. Macrophage receptors and immune recognition. Annu. Rev. Immunol. 23:901-944.

19. Hume, D.A., et al. 2002. The mononuclear phagocyte system revisited. J. Leukoc. Biol. 72:621-627.

20. Byrne, P.V., Guilbert, L.J., and Stanley, E.R. 1981. Distribution of cells bearing receptors for a colonystimulating factor (CSF-1) in murine tissues. J. Cell Biol. 91:848-853.

21. Tacke, F., et al. 2006. Immature monocytes acquire antigens from other cells in the bone marrow and present them to $T$ cells after maturing in the periphery. J. Exp. Med. 203:583-597.

22. Geissmann, F., Jung, S., and Littman, D.R. 2003. Blood monocytes consist of two principal sub- sets with distinct migratory properties. Immunity. 19:71-82.

23. Steinberg, D. 2005. Hypercholesterolemia and inflammation in atherogenesis: two sides of the same coin. Mol. Nutr. Food Res. 49:995-998.

24. Aikawa, M., et al. 2002. Lipid lowering reduces oxidative stress and endothelial cell activation in rabbit atheroma. Circulation. 106:1390-1396.

25. Takahashi, K., et al. 2003. Adiposity elevates plasma MCP-1 levels leading to the increased CD11b-positive monocytes in mice. J. Biol. Chem. 278:46654-46660.

26. Huang, Z.S., Chien, K.L., Yang, C.Y., Tsai, K.S., and Wang, C.H. 2001. Peripheral differential leukocyte counts in humans vary with hyperlipidemia, smoking, and body mass index. Lipids. 36:237-245.

27. Jagels, M.A., Chambers, J.D., Arfors, K.E., and Hugli, T.E. 1995. C5a- and tumor necrosis factor-alphainduced leukocytosis occurs independently of beta 2 integrins and L-selectin: differential effects on neutrophil adhesion molecule expression in vivo. Blood. 85:2900-2909.

28. Terashima, T., English, D., Hogg, J.C., and van Eeden, S.F. 1998. Release of polymorphonuclear leukocytes from the bone marrow by interleukin-8. Blood. 92:1062-1069.

29. Martin, C., et al. 2003. Chemokines acting via CXCR2 and CXCR4 control the release of neutrophils from the bone marrow and their return following senescence. Immunity. 19:583-593.

30. Suratt, B.T., et al. 2004. Role of the CXCR4/SDF-1 chemokine axis in circulating neutrophil homeostasis. Blood. 104:565-571.

31. Daly, C., and Rollins, B.J. 2003. Monocyte chemoattractant protein-1 (CCL2) in inflammatory disease and adaptive immunity: therapeutic opportunities and controversies. Microcirculation. 10:247-257.

32. Serbina, N.V., and Pamer, E.G. 2006. Monocyte emigration from bone marrow during bacterial infection requires signals mediated by chemokine receptor CCR2. Nat. Immunol. 7:311-317.

33. Mack, M., et al. 2001. Expression and characterization of the chemokine receptors CCR2 and CCR5 in mice. J. Immunol. 166:4697-4704. 